\title{
Treated bacterial endocarditis as a histological mimic of fungal infection
}

\author{
D E Roskell, I C J W Bowler, P Barnes
}

\begin{abstract}
Histological assessment of cardiac valve tissue contributes to the diagnosis of infective endocarditis and is of particular importance in cases in which no organism is cultured. Antibiotic treatment of bacterial endocarditis may lead to abnormal bacterial morphology and staining characteristics. Although in many cases the presence of some residual bacteria of normal appearance makes the diagnosis straightforward, in some only abnormal bacteria may be seen. Unless the appearances of these are interpreted with caution, the presence of larger spherical organisms with the staining properties of a yeast may lead to an erroneous diagnosis of fungal infection.

(F Clin Pathol 1998;51:539-540)
\end{abstract}

Keywords: infective endocarditis; fungal infection; misdiagnosis

\section{Case report}

A 64 year old man underwent aortic valve replacement for aortic incompetence. During the previous months he had developed clinical signs and symptoms of endocarditis. Blood cultures had grown Streptococcus sanguis, and he had been treated with a prolonged course of antibiotics (14 days of penicillin and gentamicin followed by 14 days of ceftriaxone) before surgery. Parts of the excised valve were sent for histological and microbiological assessment.

The valve leaflet sent for histology appeared thickened with an irregular surface, particularly towards its free edge. Histology confirmed thickening of the cusp with fibrosis and vascularisation, and the presence on one surface of a fibrinous vegetation. Beneath this there was a histiocytic infiltrate, containing numerous foamy macrophages and giant cells, and scattered lymphocytes. No neutrophils were seen, although there was some necrosis associated with the chronic inflammation. Haematoxylin and eosin sections showed the presence of spherical organisms within the vegetation, their size and shape suggesting a yeast rather than a bacterial infection.

Grocott silver and periodic acid Schiff (PAS) stained the organisms, but there was no Gram positive staining despite repeated tests with appropriate controls (fig 1). On the grounds of the morphology of the organism and the rather granulomatous inflammation, the possibility of a fungal (probably Candida $\mathrm{sp}$ ) endocarditis
John Radcliffe

Hospital, Oxford, UK Nuffield Department of Pathology and Bacteriology D E Roskell

Department of Microbiology and Oxford Public Health

Laboratory

I C J W Bowler

P Barnes

Correspondence to Dr Roskell, Oxford University Nuffield Department of Pathology and Bacteriology, John Radcliffe Hospital, Oxford OX3 9DU, UK.

Accepted for publication 11 March 1998
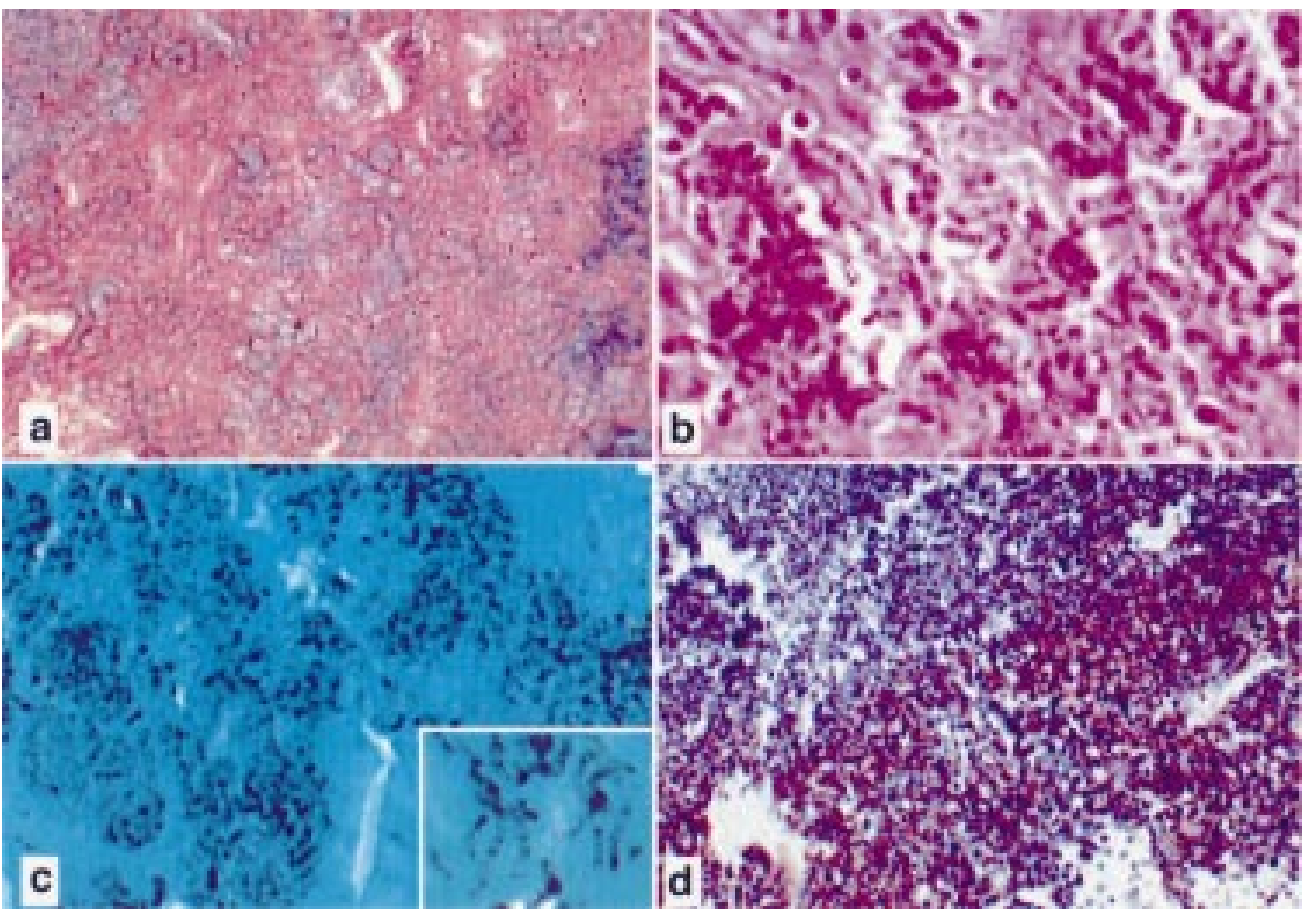

Figure 1 High power photographs of streptococci in vegetations of endocarditis: (a) haematoxylin and eosin; (b) PAS; (c) Grocott silver stains of a case with enlarged cocci which did not stain with Gram stain; (d) another case of endocarditis showing conventional streptococci staining Gram positive. All photographs are shown at the same magnification $(\times 100$ objective under oil). 
was considered. However, the microbiological specimen was sterile despite prolonged fungal culture.

Close inspection of the histology showed that, while the spherical organisms were of a size consistent with candida, no hyphae or convincing budding could be demonstrated. In many areas, chains of spheres were seen. It was concluded that the appearances were most likely to represent the streptococcus which had been isolated previously from the patient's blood cultures and that the unusual morphology and staining resulted from the effects of antibiotic treatment.

No further antimicrobial treatment was given, and the patient made an uncomplicated recovery with no further episodes of infection.

\section{REVIEW OF FURTHER CASES}

To assess the prevalence of this phenomenon, the histology of nine recent cases of endocarditis caused by Gram positive cocci (staphylococci or streptococci) was reviewed.

Six of these contained easily visible Gram positive cocci and presented no diagnostic difficulty. One case (described above) contained only enlarged Grocott positive cocci. Two cases contained predominantly enlarged Grocott positive cocci mixed with occasional Gram positive cocci. Grocott staining of the cases with obvious Gram positive cocci showed that these too contained occasional enlarged Grocott positive cocci.

\section{Discussion}

Histological assessment of cardiac valve tissue contributes to the diagnosis of infective endocarditis and is of particular importance in cases where no organism is cultured. ${ }^{1}$ A significant number of patients undergo surgical treatment for infective endocarditis without a definitive preoperative microbiological diagnosis. Many of these have been treated with empirical antibiotics, and no organism is grown from the excised tissue. ${ }^{2}$ This leaves histological assessment to confirm the diagnosis of infection. A misdiagnosis of fungal endocarditis could lead to unnecessary, expensive, and damaging treatment with an antifungal agent in a patient who has already effectively been cured. Fungal endocarditis is rare, usually occurring in the context of immunosuppression and prolonged intravenous cannulation, or intravenous drug abuse. None of the patients in this study had such risk factors.

It is known that antibiotic treatment can produce abnormal bacterial morphology, both in vivo and in vitro, ${ }^{3}{ }^{4}$ including in vitro growth of giant staphylococci induced by subinhibitory concentrations of antibiotics. ${ }^{3}$ When undertaking histological assessment of a valve with endocarditis the effects of antibiotic treatment must be considered. Gram staining depends on a reaction with the bacterial cell wall, and it is this which is targeted by many antibiotics in routine use. Antibiotic treatment may disrupt the cell wall so that a bacterium loses its characteristic Gram stain and its morphology. In situations where such probably dead organisms are accessible to phagocytes they will not be seen in significant numbers in histological sections. However, in areas which are relatively inaccessible to phagocytes, such as in cases of bacterial endocarditis, enlarged dead bacteria with unusual staining characteristics may mimic fungal infection and lead to diagnostic confusion.

1 Durack DT, Lukes AS, Bright DK, the Duke Endocarditis Service. New criteria for the diagnosis of infective endocarditis: utilisation of specific echocardiographic findings. Am $\mathcal{f}$ Med 1994;96:200-9.

2 Hoen B, Selton Suty C, Lacassin F, et al. Infective endocarditis in patients with negative blood cultures: analysis of 88 cases from a one-year nationwide survey in France. Clin Infect Dis 1995;20:501-6.

3 Lorian V, Atkinson B. Abnormal forms of bacteria produced by antibiotics. Am $\mathcal{F}$ Clin Pathol 1975;64:678-88.

4 Frehel C, Hellio R, Cremieux AC, et al. Nutritionally variant streptococci develop ultrastructural abnormalities during experimental endocarditis. Microb Pathog 1988;4:247-55. 